\title{
PATTERNS OF TRAUMATIC INJURIES AND MORTALITY IN TERTIARY TRAUMA CENTER, ASSIUT UNIVERSITY HOSPITALS (FROM 2005 TO 2012)
}

\author{
Saly Y Abdel-Hameed ${ }^{1}$, Ahmed K Ibrahim ${ }^{2}$, , Hayam Z Thabet ${ }^{1}$ and Aml A Mohamed ${ }^{1}$ \\ ${ }^{1}$ Department of Forensic medicine and Clinical Toxicology, Faculty of Medicine, Assiut \\ University, Assiut, Egypt, ${ }^{2}$ Department of Public Health \& Community Medicine, Faculty of \\ Medicine, Assiut University, Assiut, Egypt \\ *Correspondence: Saly Y Abdel-Hameed, Department of Forensic medicine and Clinical \\ Toxicology, Faculty of Medicine, Assiut University, Assiut, EgyptE-mail: \\ saso8765@yahoo.com
}

\begin{abstract}
Trauma is defined as any harm occurs to the human body due to application of force. Every minute in life we are liable to different forms of trauma. Traumatic injuries are one of the leading causes for death and infirmities. There is increasing rate of exposure to trauma especially in developing countries. This raises the concern for documentation and analysis of the causes to plan preventive strategy to decrease the mortality and morbidity.

Aim of the study: to cast light on the pattern of trauma and mortality causes, patients at risk and the value of documentation in medicolegal work and in helping the authorities identifying the changes in causes of trauma pattern helping in building preventive measures

Subjects and methods: Descriptive retrograde study done on patients admitted to tertiary trauma center in Assiut University Hospitals (AUH) in the period from 20052012. Analysis of the demographic data, cause and site of injury and mortality cases were carried out.

Results: 77,380 patients were admitted during the period of the study. Age mean \pm SD was $29.41 \pm 20.8$ years, males were more predominant $75.5 \%$ compared to $24.5 \%$ females. The improvement was $78.9 \%$ in admitted patients and the death rate was $4 \%$. The top causes of injury and mortality were; falling on the ground, road traffic accidents, falling from height, interpersonal violence and blunt injuries. The most affected body site was the lower limbs, upper limbs, head, vertebral column, chest, pelvis followed by abdomen. Conclusion: the road traffic accidents, falling accidents and interpersonal violence were the main causes of mortality. Attention must be paid for the young patients exposed to these injuries especially if head, lower limb, chest or abdomen were affected as they were at increasing risk. Also, Attention must be paid for documentation of injuries in details due to its medicolegal value.
\end{abstract}

Keywords: traumatic injuries, mortality, forensic medicine, documentation, Assiut, Egypt

\section{INTRODUCTION}

Injuries and violence are threats to health in every country of the world. They account for $9 \%$ of global mortality- more than five million deaths every year (World Health Organization, 2007). Injury is the physical damage when a human body is 
subjected to intolerable levels of energy. It may be intentional deliberate, unintentional accidental or undetermined intent (Holder et al., 2001).

Accelerated urbanization and industrialization during the last four decades lead to increasing rate of traumatic injuries and violence (Dsouza, 2014). In the Eastern Mediterranean Region, more than 400, 000 people die from different causes of injuries every year (World Health Organization, 2010).

The emergency department (ED) is a unique dynamic environment, where overwhelming number of patients seen (Kovacs and Croskerry, 1999). Such busy environment, where decisions are made under time pressure and with incomplete information, have been considered conducive to errors and claims of malpractice ( $\mathbf{Y u}$ and Green, 2009).

Clinical forensic medicine (CFM) is "the application of appropriate forensic practices and principles, to living patients in a clinical setting. This is including the survivors of trauma and potentially catastrophic experiences resulting in injury (Recktenwald et al., 2005). It has been increasingly recognized as playing a crucial role in trauma care (Sharma, 2006). Injuryrelated data are essential elements in surveillance, and ED records provide an important source of information (Runyan et al., 1992). Appropriate medical documentation and interpretation of physical findings may aid law enforcement and/or social services in the legal evaluation of a case or situation (Recktenwald et al., 2005) Also, this may protect physicians from liability during a malpractice suit (Wegman et al., 2012).

\section{THE AIM OF THE STUDY}

This study aimed to cast light on the pattern of trauma and mortality causes, patients at risk and the value of documentation in medicolegal work and in helping the authorities identifying the changes in causes of trauma pattern helping in building preventive measures.

\section{SUBJECTS \& METHODS}

Assiut University Hospital (AUH) is a big referral hospital for all Upper Egypt governorates. The trauma unit in AUH is a tertiary level unit receiving all types of trauma, except cases of burn, either direct or referred from other hospitals. The trauma unit admits patients to undergo operative treatment or admitted to the intensive care in trauma unit but patients who visited the unit for minor injuries which did not need admission were not included in the database of the trauma unit.

The data of admitted cases to trauma unit during the period (20052012) were collected from the data base system in AUH after approval from the hospital authority and ethical committee in the Faculty of Medicine, Assiut University.

After this period, from 2013 up till now the data base was not involved in our study because; new regulations limited the admission to AUH to three days a week only so affecting the rate admission.

The data included; the demographic data (age, sex, residence and occupation), cause of injury, body site of the injury, death rate, duration of 
stay in the hospital and incidence rate of trauma cases admitted to trauma unit, AUH from Assiut governorate 2005-2012

The terms used to describe causes of injuries were the same as recorded in the data base system in AUH.

\section{Statistical Analysis}

Data was reviewed for accuracy and completeness; duplicates and incomplete sheets were removed. Data was coded then SPSS software version 20 (SPSS Inc., Chicago, IL, USA) was used for data processing. Descriptive statistics (frequencies) were used to describe the characteristics of the studied cohort. The relationships were tested using chi-square analysis $(\chi 2)$. A p-value of $\leq 0.05$ was considered statistically significant.

\section{RESULTS}

The total number of patients admitted to the trauma unit in the period of the study from 2005 till 2012 was 77,380 patients.

Table (1) describes the demographic data for the admitted patients;

- Most of the patients were males representing about three quarters of the total number $75.5 \%(58,418)$ and the females were $24.5 \%(18,962)$.

- The age ranged from (0-105 year) and mean \pm SD $(29.4 \pm 20.8)$. Patients in the second decade represented $21.4 \%$ of patients, followed by patients $<10$ years old $(20 \%)$, while the least percentage $(5.9 \%)$ was of patients > 70 years (fig.1).

- Assiut governorate residents were $70.8 \%(54,808)$ and $29.1 \%(22,543)$ from outside the governorate. Unemployed patients constituted $60.1 \%(46,517)$ of the patients. -There was improvement in $78.9 \%$ (61.072). Trauma was responsible for mortality in $4 \%(3,172)$ of patients.

Also, table (1) showing that the leading cause of injury in $30.4 \%$ $(23,534)$ patients was road traffic accidents (RTA) followed by fall on the ground (FG) in $28.1 \%(21,750)$, while the least causes were machine injury (MI) in $2.1 \%(1,598)$ and animal bite (AB) in 1\% (754) (fig.2). The duration of stay in hospital was mean \pm SD $4.57 \pm 6.1$ days.

Analysis of the mortality determinant is shown in table (2). Total patients died after admission to trauma center was 3127 which represents $4 \%$ of the total admitted patients during the study period. The age was $33.32 \pm 25.3$ (mean $\pm \mathrm{SD}$ ), male patients were more predominant than females $(68.3 \%$ vs. $31.7 \%)$. The first cause of death was the road traffic accidents $40.2 \%$ followed by fall on the ground $23.8 \%$ (fig.3). The range of stay in hospital was 1-184 days with mean \pm SD 6.21 \pm 10.4 .

Table (3) demonstrates the difference in pattern of injuries in each year of the studied period. The fall on ground, road traffic accidents and fall from height were the first three causes of admission along the duration of the study. The firearm injuries appeared as the fourth cause of admission in 2012.

In table (4) shows the leading causes of death in admitted patients confined to fall on the ground, road traffic accidents, fall from height, interpersonal violence and blunt object injuries. Firearm injuries began to rise to be the fourth cause of death in 2011 and the second cause of death in 2012.

Chi-square test analysis to examine the relation between Age group and 
cause of Trauma revealed the presence of significant distribution of age groups in admitted cases in relation to the cause of injury. Falling from height followed by falling on ground, animal bite and blunt object and sharp instrument injuries are the predominant causes of injury in the first decade of life. In the second decade, all injuries occurred in similar rate. Firearm injuries are the commonest in the third and fourth decade. Interpersonal violence was the predominant cause of trauma in patients in the fifth and sixth decade. Fall on the ground was the main cause of trauma in patients aged above 60 years (table 5).

Table (6) shows that firearm injuries was the most common cause of death in cases $\leq 10$ years old followed by falling from height, machine injury and road traffic accidents. In the second decade deaths occurs more with animal bite injuries.

Incidence rate of traumatic injuries in Assiut Governorate residents admitted to AUH was highest in 2009 followed by 2008 the least incidence rate was in 2012 (table 7 and fig. 4).
The highest cause- specific mortality rate was for firearm and road traffic injuries in studied cases (table 8 and fig.5).

The commonest body areas injured in the admitted cases to the trauma unit in AUH were; The lower limb, upper limb and head (26.0, 23.4 and $16.4 \%$, respectively) (table 9 and fig.6).

The most dangerous areas leading to death after injury shown in (fig. 7); $56.7 \%$ of total deaths was due to head injuries, followed by $20.1 \%$ by lower limb injuries then 19.9 by chest injuries.

Analysis of the mortality in relation to body part affected and cause of injury represented in (table 10). In road traffic accidents chest injuries $(65.2 \%)$, pelvis and head (62.2\%) followed by vertebral column injuries causing deaths in $56.9 \%$ of road traffic accidents deaths. Abdominal injuries causing death in $37.2 \%$ of firearm injuries deaths. Falling on the ground causing death due to lower limb injuries in $26.3 \%$ of deaths. Vertebral column injuries $(34.3 \%)$ and upper limb injuries $(29.6 \%)$ causing death in falling from height deaths. 
Table (1): Descriptive Statistics of admission to Trauma Unit, AUH, 2005-2012

\begin{tabular}{|c|c|c|}
\hline Variable & Category & $\mathbf{N}=77380$ \\
\hline \multicolumn{3}{|l|}{ Age in years } \\
\hline - $\quad$ Mean \pm SD & \multicolumn{2}{|c|}{$29.41 \pm 20.8$} \\
\hline $\begin{array}{ll}- & \text { Median }(I Q R) \\
- & \text { Range } \\
\end{array}$ & \multicolumn{2}{|c|}{$\begin{array}{c}25(33) \\
(0-105 \text { years })\end{array}$} \\
\hline \multirow{2}{*}{ Sex } & Male & $58,418(75.5 \%)$ \\
\hline & Female & $18,962(24.5 \%)$ \\
\hline \multirow{3}{*}{ Residence } & Assiut & $54,808(70.8 \%)$ \\
\hline & Outside Assiut & $22,543(29.1 \%)$ \\
\hline & Unknown & $29(0.1 \%)$ \\
\hline \multirow{3}{*}{ Occupation } & Unemployed & $46,517(60.1 \%)$ \\
\hline & Intermediate Occupation & $23,099(29.9 \%)$ \\
\hline & High Occupation & $7,764(10 \%)$ \\
\hline \multirow{4}{*}{ Outcome } & Improved & $61,072(78.9 \%)$ \\
\hline & Death & $3,172(4 \%)$ \\
\hline & Discharge on request & $6,128(8 \%)$ \\
\hline & Escape & $7,053(9.1 \%)$ \\
\hline \multirow[t]{10}{*}{ Cause of Injury } & Animal Bite (AB) & $754(1 \%)$ \\
\hline & Blunt object (BO) & $6,372(8.2 \%)$ \\
\hline & Fire Arm Injury (FAI) & $3,068(4 \%)$ \\
\hline & Fall on the Ground (FG) & $21,750(28.1 \%)$ \\
\hline & Fall from Height (FH) & $11,627(15 \%)$ \\
\hline & Inter-Personal Violence (IPV) & $6,402(8.3 \%)$ \\
\hline & Machine Injury (MI) & $1,598(2.1 \%)$ \\
\hline & Road Traffic Accident (RTA) & $23,534(30.4 \%)$ \\
\hline & Sharp instrument (SI) & $2,217(2.9 \%)$ \\
\hline & Missing & $58(0.1 \%)$ \\
\hline \multicolumn{3}{|l|}{ Hospital Stay in days } \\
\hline - $\quad$ Mean \pm SD & \multicolumn{2}{|c|}{$4.57 \pm 6.1$} \\
\hline
\end{tabular}




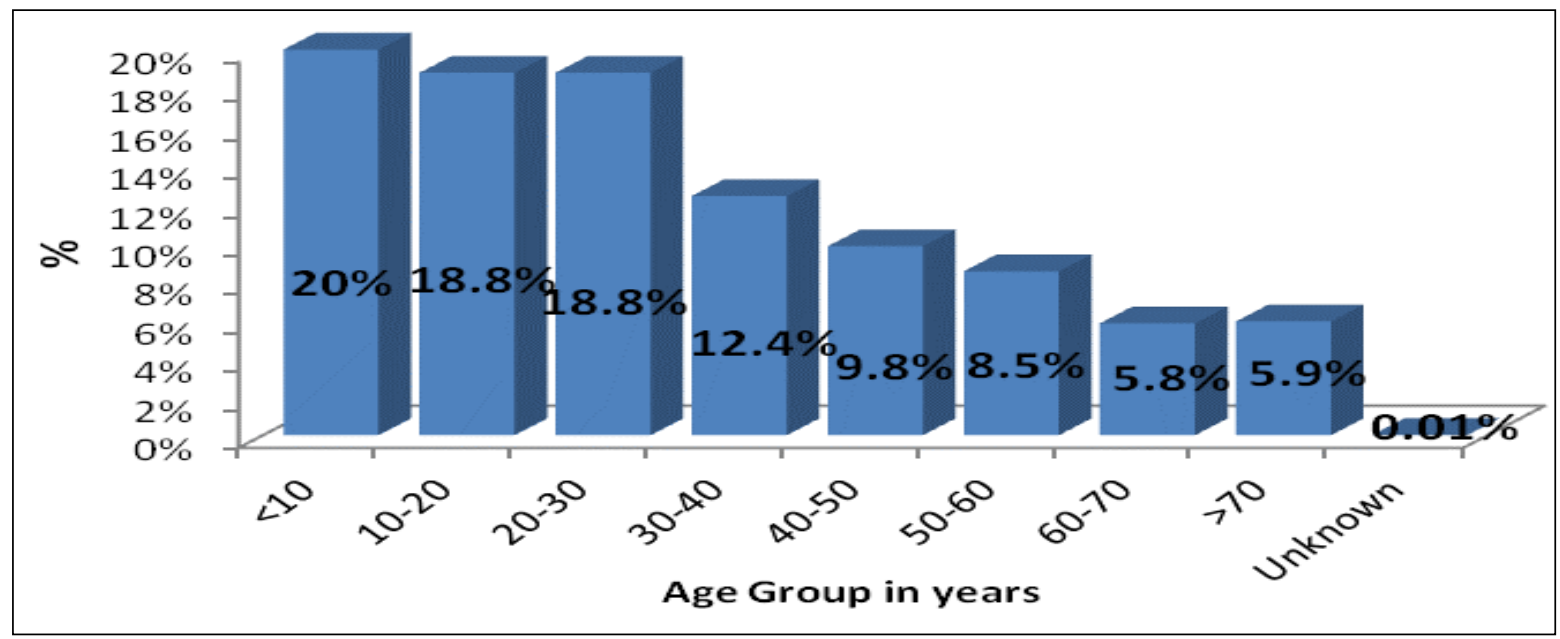

Figure (1): Age distribution of the studied patients.

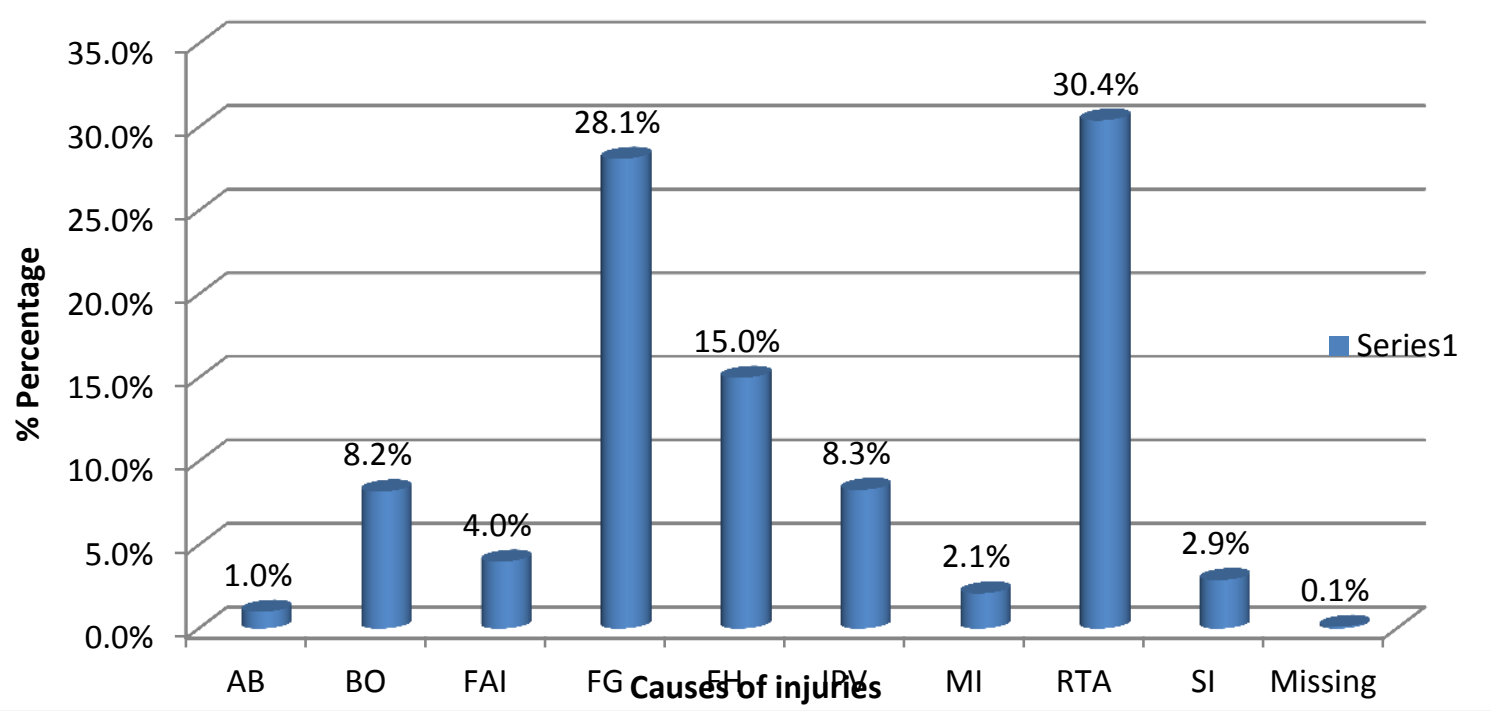

Figure (2): Causes of Injury of the admitted cases (2005-2012)

Table (2): Determinants of Mortality in patients admitted to Trauma Unit, AUH, 2005-2012

\begin{tabular}{|c|c|c|}
\hline Variable & Category & $\mathbf{N}=\mathbf{3 1 2 7}$ \\
\hline \multicolumn{3}{|l|}{ Age in years } \\
\hline - $\quad$ Mean \pm SD & \multicolumn{2}{|c|}{$33.32 \pm 25.3$} \\
\hline Median (IQR) & \multicolumn{2}{|c|}{$30(48)$} \\
\hline Range & \multicolumn{2}{|c|}{$(0-96$ years $)$} \\
\hline \multirow[t]{2}{*}{ Sex } & Male & $2,136(68.3 \%)$ \\
\hline & Female & $991(31.7 \%)$ \\
\hline \multirow[t]{3}{*}{ Residence } & Assiut & $2,174(69.5 \%)$ \\
\hline & Outside Assiut & $948(30.3 \%)$ \\
\hline & Unknown & $5(0.2 \%)$ \\
\hline \multirow[t]{3}{*}{ Occupation } & Unemployed & $2,047(65.5 \%)$ \\
\hline & Intermediate Occupation & $831(26.6 \%)$ \\
\hline & High Occupation & $249(7.9 \%)$ \\
\hline
\end{tabular}




\begin{tabular}{|l|l|c|}
\hline Cause of Injury & Animal Bite (AB) & $22(0.7 \%)$ \\
\hline & Blunt Object (BO) & $175(5.6 \%)$ \\
\hline & Fire Arm Injury (FAI) & $172(5.5 \%)$ \\
\hline & Fall on the Ground (FG) & $\mathbf{7 4 3}(\mathbf{2 3 . 8 \%})$ \\
\hline & Fall from Height (FH) & $\mathbf{4 4 0}(\mathbf{1 4 . 1 \% )}$ \\
\hline & Inter-Personal Violence (IPV) & $227(7.3 \%)$ \\
\hline & Machine Injury (MI) & $45(1.4 \%)$ \\
\hline & Road Traffic Accident (RTA) & $\mathbf{1 , 2 6 1 ( 4 0 . 2 \% )}$ \\
\hline & Sharp Instrument (SI) & $40(1.3 \%)$ \\
\hline & Unknown & $2(0.1 \%)$ \\
\hline Hospital Stay in days & \multicolumn{2}{|c|}{$\mathbf{6 . 2 1} \pm \mathbf{1 0 . 4}$} \\
\hline$\bullet$ & Mean \pm SD & \multicolumn{2}{|c|}{$\mathbf{3}$} \\
\hline & Median & $\mathbf{0 1 8 4}$ days) \\
\hline & Range & \multicolumn{2}{|c|}{} \\
\hline
\end{tabular}

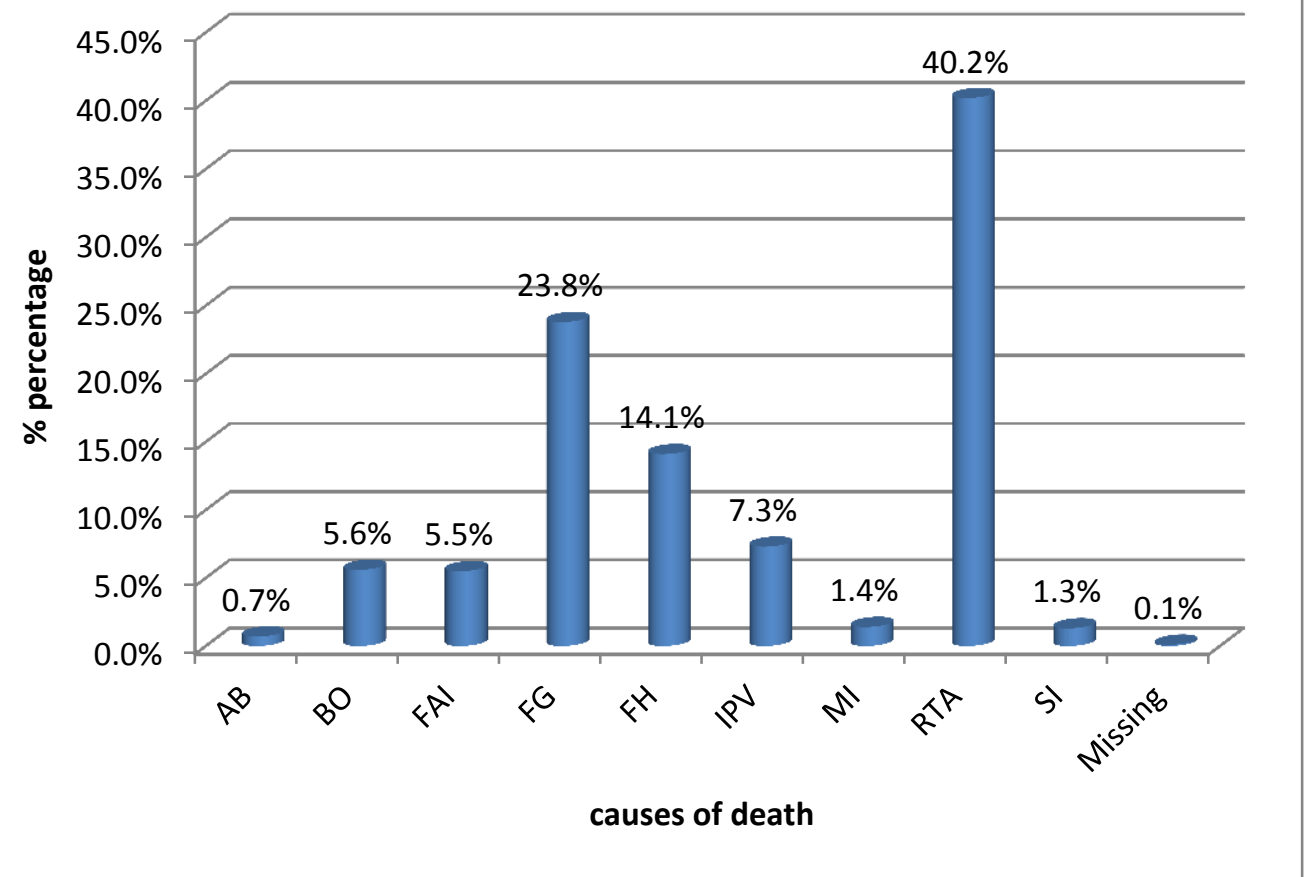

Figure (3): Percentage of deaths according to cause of injury (2005-2012) 
Table (3): The Top Five Leading Causes of Admission to Trauma Unit, AUH, 2005-2012.

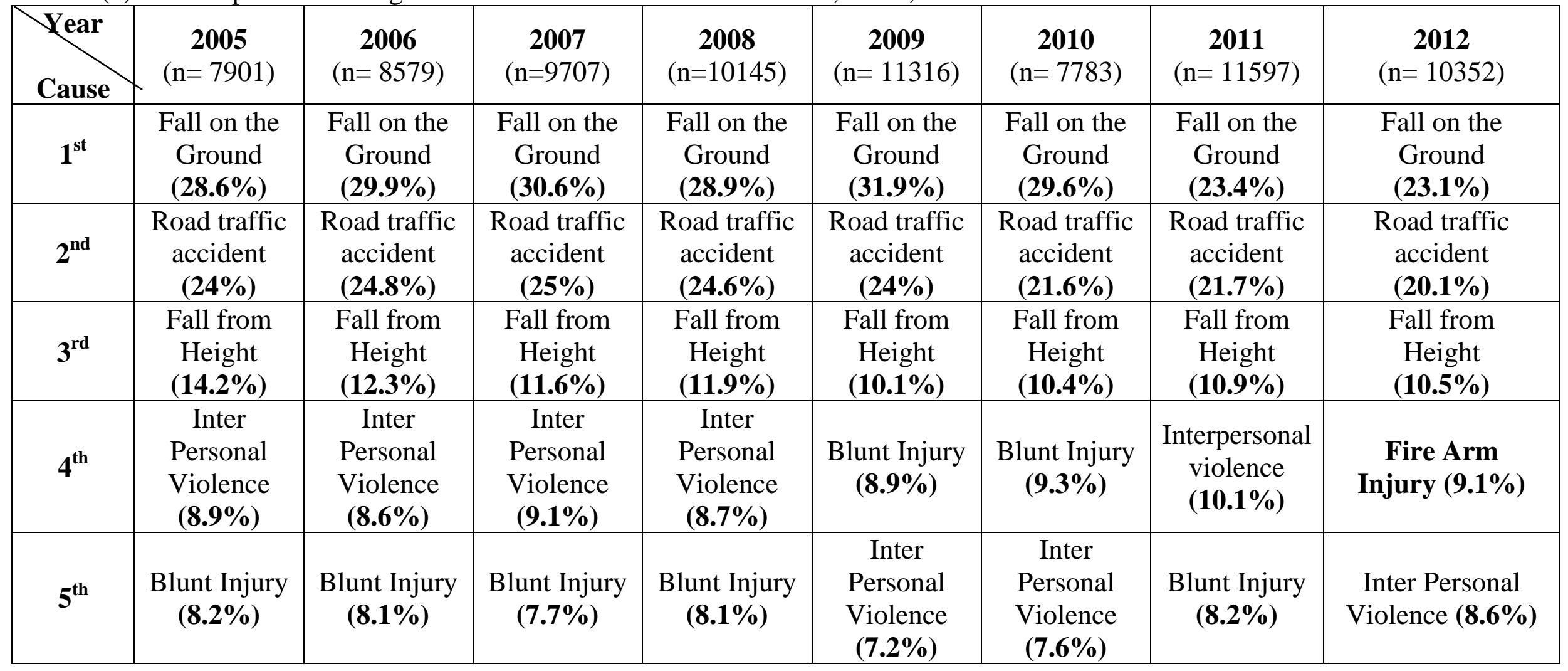


Table (4): The Top Five Leading Causes of Death in patients admitted to Trauma Unit, AUH, 2005-2012

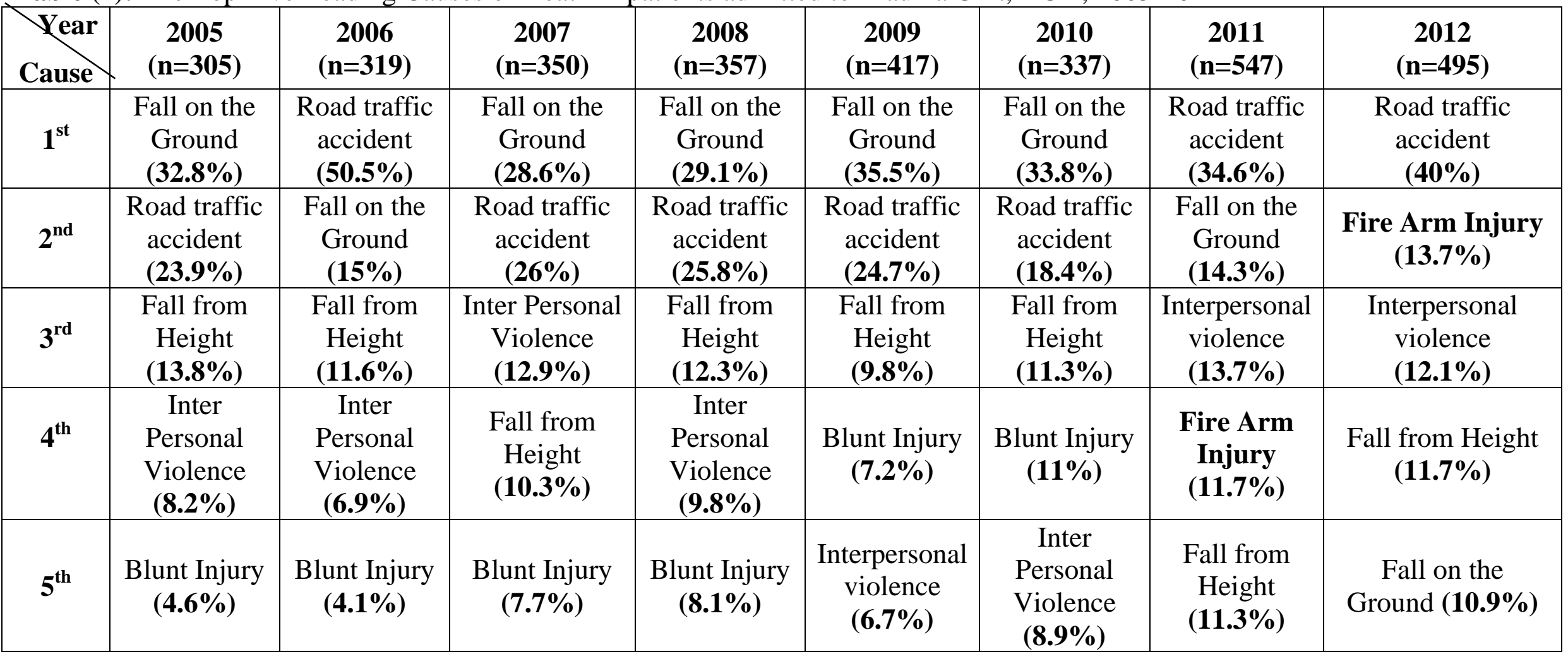


Table (5): Distribution of admissions to Trauma Unit, Assiut University Hospital, 2005-2012 in relation to cause of injury and age group:

\begin{tabular}{|c|c|c|c|c|c|c|c|c|c|c|}
\hline $\begin{array}{l}\text { Age Group } \\
\text { Cause of } \\
\text { injury }\end{array}$ & $<10$ & 11-20 & 21-30 & $31-40$ & 41-50 & $51-60$ & 61-70 & $>70$ & p-value & $\begin{array}{c}\text { Post- hoc } \\
\text { test }\end{array}$ \\
\hline $\begin{array}{l}\mathbf{A B} \\
(n=754)\end{array}$ & $\begin{array}{c}163 \\
(21.6 \%)\end{array}$ & $\begin{array}{c}172 \\
(22.8 \%)\end{array}$ & $\begin{array}{c}129 \\
(17 \%)\end{array}$ & $\begin{array}{c}66 \\
(8.8 \%)\end{array}$ & $\begin{array}{c}87 \\
(11.5 \%)\end{array}$ & $60(8 \%)$ & $\begin{array}{c}54 \\
(7.2 \%) \\
\end{array}$ & $\begin{array}{c}23 \\
(3.1 \%)\end{array}$ & \multirow[t]{9}{*}{$<0.001 * * *$} & $<0.001$ \\
\hline $\begin{array}{l}\text { BO } \\
(n=6372)\end{array}$ & $\begin{array}{c}1321 \\
(20.7 \%)\end{array}$ & $\begin{array}{c}1455 \\
(22.8 \%)\end{array}$ & $\begin{array}{l}1276 \\
(20 \%)\end{array}$ & $\begin{array}{c}783 \\
(12.3 \%)\end{array}$ & $\begin{array}{c}607 \\
(9.5 \%)\end{array}$ & $\begin{array}{c}432 \\
(6.8 \%) \\
\end{array}$ & $\begin{array}{c}296 \\
(4.6 \%) \\
\end{array}$ & $\begin{array}{c}202 \\
(3.3 \%)\end{array}$ & & $<0.001$ \\
\hline $\begin{array}{l}\text { FAI } \\
(\mathrm{n}=3068)\end{array}$ & $\begin{array}{c}376 \\
(12.3 \%)\end{array}$ & $\begin{array}{c}680 \\
(22.2 \%)\end{array}$ & $\begin{array}{c}745 \\
(24.3 \%)\end{array}$ & $\begin{array}{c}446 \\
(14.5 \%)\end{array}$ & $\begin{array}{c}332 \\
(10.7 \%)\end{array}$ & $\begin{array}{c}243 \\
(7.9 \%)\end{array}$ & $\begin{array}{c}146 \\
(4.8 \%)\end{array}$ & $\begin{array}{c}100 \\
(3.3 \%)\end{array}$ & & $<0.001$ \\
\hline $\begin{array}{l}\mathbf{F G} \\
(n=21750)\end{array}$ & $\begin{array}{c}4725 \\
(21.7 \%)\end{array}$ & $\begin{array}{c}4356 \\
(20 \%)\end{array}$ & $\begin{array}{c}3572 \\
(16.4 \%)\end{array}$ & $\begin{array}{c}2353 \\
(10.8 \%)\end{array}$ & $\begin{array}{c}2092 \\
(9.6 \%)\end{array}$ & $\begin{array}{l}1828 \\
(8.4 \%)\end{array}$ & $\begin{array}{l}1514 \\
(7 \%)\end{array}$ & $\begin{array}{l}1308 \\
(6 \%)\end{array}$ & & $<0.001$ \\
\hline $\begin{array}{l}\mathbf{F H} \\
(n=11627)\end{array}$ & $\begin{array}{c}2789 \\
(24 \%)\end{array}$ & $\begin{array}{c}2418 \\
(20.8 \%)\end{array}$ & $\begin{array}{c}2122 \\
(18.3 \%)\end{array}$ & $\begin{array}{c}1283 \\
(11 \%)\end{array}$ & $\begin{array}{c}1133 \\
(9.7 \%)\end{array}$ & $\begin{array}{c}885 \\
(7.6 \%)\end{array}$ & $\begin{array}{c}572 \\
(4.9 \%)\end{array}$ & $\begin{array}{c}425 \\
(3.7 \%)\end{array}$ & & $=0.001$ \\
\hline $\begin{array}{l}\text { IPV } \\
(n=6402)\end{array}$ & $\begin{array}{c}880 \\
(13.8 \%)\end{array}$ & $\begin{array}{c}1365 \\
(21.3 \%)\end{array}$ & $\begin{array}{c}1398 \\
(21.8 \%)\end{array}$ & $\begin{array}{c}899 \\
(14 \%)\end{array}$ & $\begin{array}{c}741 \\
(11.6 \%)\end{array}$ & $\begin{array}{c}548 \\
(8.6 \%)\end{array}$ & $\begin{array}{c}334 \\
(5.2 \%)\end{array}$ & $\begin{array}{c}235 \\
(3.7 \%)\end{array}$ & & $=0.001$ \\
\hline $\begin{array}{l}\text { MI } \\
(n=1598)\end{array}$ & $\begin{array}{c}301 \\
(18.8 \%) \\
\end{array}$ & $\begin{array}{c}353 \\
(22.1 \%) \\
\end{array}$ & $\begin{array}{c}328 \\
(20.5 \%) \\
\end{array}$ & $\begin{array}{c}201 \\
(12.6 \%) \\
\end{array}$ & $\begin{array}{c}160 \\
(10 \%) \\
\end{array}$ & $\begin{array}{c}111 \\
(6.9 \%) \\
\end{array}$ & $\begin{array}{c}87 \\
(5.4 \%) \\
\end{array}$ & $\begin{array}{c}57 \\
(3.6 \%)\end{array}$ & & $=0.001$ \\
\hline $\begin{array}{l}-\quad \text { RTA } \\
(n=23534)\end{array}$ & $\begin{array}{c}4388 \\
(18.6 \%)\end{array}$ & $\begin{array}{c}5315 \\
(22.6 \%)\end{array}$ & $\begin{array}{c}4645 \\
(19.7 \%)\end{array}$ & $\begin{array}{c}3053 \\
(13 \%)\end{array}$ & $\begin{array}{c}2404 \\
(10.2 \%)\end{array}$ & $\begin{array}{c}1747 \\
(7.4 \%)\end{array}$ & $\begin{array}{l}1182 \\
(5 \%)\end{array}$ & $\begin{array}{c}797 \\
(3.4 \%) \\
\end{array}$ & & $=0.001$ \\
\hline $\begin{array}{lr}-\quad \text { SI } \\
(\mathrm{n}=2217)\end{array}$ & $\begin{array}{c}482 \\
(21.7 \%) \\
\end{array}$ & $\begin{array}{c}463 \\
(20.9 \%) \\
\end{array}$ & $\begin{array}{c}490 \\
(22.1 \%) \\
\end{array}$ & $\begin{array}{c}285 \\
(12.9 \%) \\
\end{array}$ & $\begin{array}{c}205 \\
(9.2 \%) \\
\end{array}$ & $\begin{array}{c}135 \\
(6.1 \%) \\
\end{array}$ & $\begin{array}{c}90 \\
(4.1 \%)\end{array}$ & $\begin{array}{c}67 \\
(3 \%) \\
\end{array}$ & & $=0.017$ \\
\hline
\end{tabular}

*Chi-square test analysis was used to examine the association between Age group and Type of Trauma $\mathbf{p}<0.001$ is very highly significance 
Table (6): Death Frequency among patients admitted to Trauma Unit, AUH, 2005-2012 in relation to Age Group and Cause of Trauma.

\begin{tabular}{|c|c|c|c|c|c|c|c|c|c|c|}
\hline $\begin{array}{l}\text { Age Group } \\
\text { Cause } \\
\text { of Trauma }\end{array}$ & $<10$ & $11-20$ & 21-30 & $31-40$ & $41-50$ & 51-60 & $61-70$ & $>70$ & p-value & Post- hoc \\
\hline $\begin{array}{l}\mathbf{A B} \\
(\mathrm{n}=22)\end{array}$ & $\begin{array}{c}4 \\
(18.2 \%)\end{array}$ & $\begin{array}{c}4 \\
(18.2 \%)\end{array}$ & $\begin{array}{c}2 \\
(9.1 \%)\end{array}$ & $0(0 \%)$ & $\begin{array}{c}4 \\
(18.2 \%)\end{array}$ & $\begin{array}{c}1 \\
(4.5 \%)\end{array}$ & $\begin{array}{c}6 \\
(27.3 \%)\end{array}$ & $\begin{array}{c}1 \\
(4.5 \%)\end{array}$ & \multirow[t]{9}{*}{$<0.001 * * *$} & $<0.001$ \\
\hline $\begin{array}{l}\mathbf{B O} \\
(n=175)\end{array}$ & $\begin{array}{c}35 \\
(20 \%)\end{array}$ & $\begin{array}{c}24 \\
(13.7 \%)\end{array}$ & $\begin{array}{c}23 \\
(13.1 \%)\end{array}$ & $\begin{array}{c}24 \\
(13.7 \%)\end{array}$ & $\begin{array}{c}15 \\
(8.6 \%)\end{array}$ & $\begin{array}{c}16 \\
(9.1 \%) \\
\end{array}$ & $\begin{array}{c}25 \\
(14.3 \%)\end{array}$ & $\begin{array}{c}13 \\
(7.4 \%)\end{array}$ & & $<0.001$ \\
\hline $\begin{array}{l}\text { FAI } \\
(\mathrm{n}=172)\end{array}$ & $\begin{array}{c}66 \\
(38.4 \%) \\
\end{array}$ & $\begin{array}{c}22 \\
(12.8 \%)\end{array}$ & $\begin{array}{c}20 \\
(11.6 \%)\end{array}$ & $\begin{array}{c}22 \\
(12.8 \%)\end{array}$ & $\begin{array}{c}20 \\
(11.6 \%)\end{array}$ & $7(4.1 \%)$ & $\begin{array}{c}11 \\
(6.4 \%)\end{array}$ & $\begin{array}{c}4 \\
(2.3 \%) \\
\end{array}$ & & $<0.001$ \\
\hline $\begin{array}{l}\mathbf{F G} \\
(\mathrm{n}=743)\end{array}$ & $\begin{array}{c}148 \\
(19.9 \%)\end{array}$ & $\begin{array}{c}72 \\
(9.7 \%)\end{array}$ & $\begin{array}{c}90 \\
(12.1 \%)\end{array}$ & $\begin{array}{c}82 \\
(11 \%)\end{array}$ & $\begin{array}{c}70 \\
(9.4 \%)\end{array}$ & $\begin{array}{c}71 \\
(9.6 \%)\end{array}$ & $\begin{array}{c}107 \\
(14.4 \%)\end{array}$ & $\begin{array}{c}103 \\
(13.4 \%)\end{array}$ & & $<0.001$ \\
\hline $\begin{array}{l}\mathbf{F H} \\
(n=440)\end{array}$ & $\begin{array}{c}163 \\
(37 \%)\end{array}$ & $\begin{array}{c}49 \\
(11.1 \%)\end{array}$ & $\begin{array}{c}46 \\
(10.5 \%)\end{array}$ & $\begin{array}{c}27 \\
(6.1 \%) \\
\end{array}$ & $\begin{array}{c}37 \\
(8.4 \%)\end{array}$ & $\begin{array}{c}45 \\
(10.2 \%)\end{array}$ & $\begin{array}{c}39 \\
(8.9 \%)\end{array}$ & $\begin{array}{c}34 \\
(7.7 \%)\end{array}$ & & $=0.001$ \\
\hline $\begin{array}{l}\text { IPV } \\
(n=227)\end{array}$ & $\begin{array}{c}43 \\
(18.9 \%) \\
\end{array}$ & $\begin{array}{c}28 \\
(12.3 \%) \\
\end{array}$ & $\begin{array}{c}33 \\
(14.5 \%) \\
\end{array}$ & $\begin{array}{c}18 \\
(7.9 \%) \\
\end{array}$ & $\begin{array}{c}28 \\
(12.3 \%) \\
\end{array}$ & $\begin{array}{c}40 \\
(17.6 \%) \\
\end{array}$ & $\begin{array}{c}23 \\
(10.1 \%) \\
\end{array}$ & $\begin{array}{c}14 \\
(6.2 \%) \\
\end{array}$ & & $=0.001$ \\
\hline $\begin{array}{l}\text { MI } \\
(n=45)\end{array}$ & $\begin{array}{c}14 \\
(31.1 \%)\end{array}$ & $\begin{array}{c}4 \\
(8.9 \%)\end{array}$ & $\begin{array}{c}6 \\
(13.3 \%)\end{array}$ & $\begin{array}{c}3 \\
(6.7 \%)\end{array}$ & $\begin{array}{c}5 \\
(11.1 \%)\end{array}$ & $\begin{array}{c}6 \\
(13.3 \%)\end{array}$ & $\begin{array}{c}4 \\
(8.9 \%)\end{array}$ & $\begin{array}{c}3 \\
(6.7 \%)\end{array}$ & & $<0.001$ \\
\hline $\begin{array}{l}\text { RTA } \\
(n=1261)\end{array}$ & $\begin{array}{c}373 \\
(29.6 \%) \\
\end{array}$ & $\begin{array}{c}165 \\
(13.1 \%) \\
\end{array}$ & $\begin{array}{c}176 \\
(14 \%) \\
\end{array}$ & $\begin{array}{c}117 \\
(9.3 \%) \\
\end{array}$ & $\begin{array}{c}133 \\
(10.6 \%) \\
\end{array}$ & $\begin{array}{c}114 \\
(9 \%) \\
\end{array}$ & $\begin{array}{c}97 \\
(7.7 \%) \\
\end{array}$ & $\begin{array}{c}85 \\
(6.7 \%) \\
\end{array}$ & & $=0.001$ \\
\hline $\begin{array}{l}\text { SI } \\
(\mathrm{n}=40)\end{array}$ & $\begin{array}{c}5 \\
(12.5 \%) \\
\end{array}$ & $\begin{array}{c}7 \\
(17.5 \%) \\
\end{array}$ & $4(10 \%)$ & $2(5 \%)$ & $8(20 \%)$ & $4(10 \%)$ & $\begin{array}{c}5 \\
(12.5 \%) \\
\end{array}$ & $\begin{array}{c}5 \\
(12.5 \%) \\
\end{array}$ & & $<0.001$ \\
\hline
\end{tabular}

*Chi-square test analysis was used to examine the association between Age group and cause of Trauma. ${ }^{* * * *} \mathbf{p}<0.001$ is very highly significance 
Table (7): Incidence Rate of Trauma cases admitted to Trauma Unit, AUH from Assiut Governorate 2005-2012

\begin{tabular}{|c|c|c|c|c|}
\hline \multicolumn{2}{|c|}{ Year } & MYP* & No. of Cases & Incidence Rate \\
\hline - & 2005 & $3,380,689$ & 5,988 & $177 / 100,000$ \\
\hline - & 2006 & $3,444,967$ & 6,459 & $187 / 100,000$ \\
\hline 一 & 2007 & $3,509,245$ & 7,250 & $207 / 100,000$ \\
\hline - & 2008 & $3,573,523$ & 7,429 & $208 / 100,000$ \\
\hline - & 2009 & $3,637,802$ & 8,247 & $227 / 100,000$ \\
\hline 一 & 2010 & $3,702,080$ & 5,373 & $145 / 100,000$ \\
\hline - & 2011 & $3,766,358$ & 7,521 & $200 / 100,000$ \\
\hline - & 2012 & $3,830,636$ & 6,541 & $171 / 100,000$ \\
\hline
\end{tabular}

*Mid-year Population of Assiut Governorate 2006 according to CAPMAS

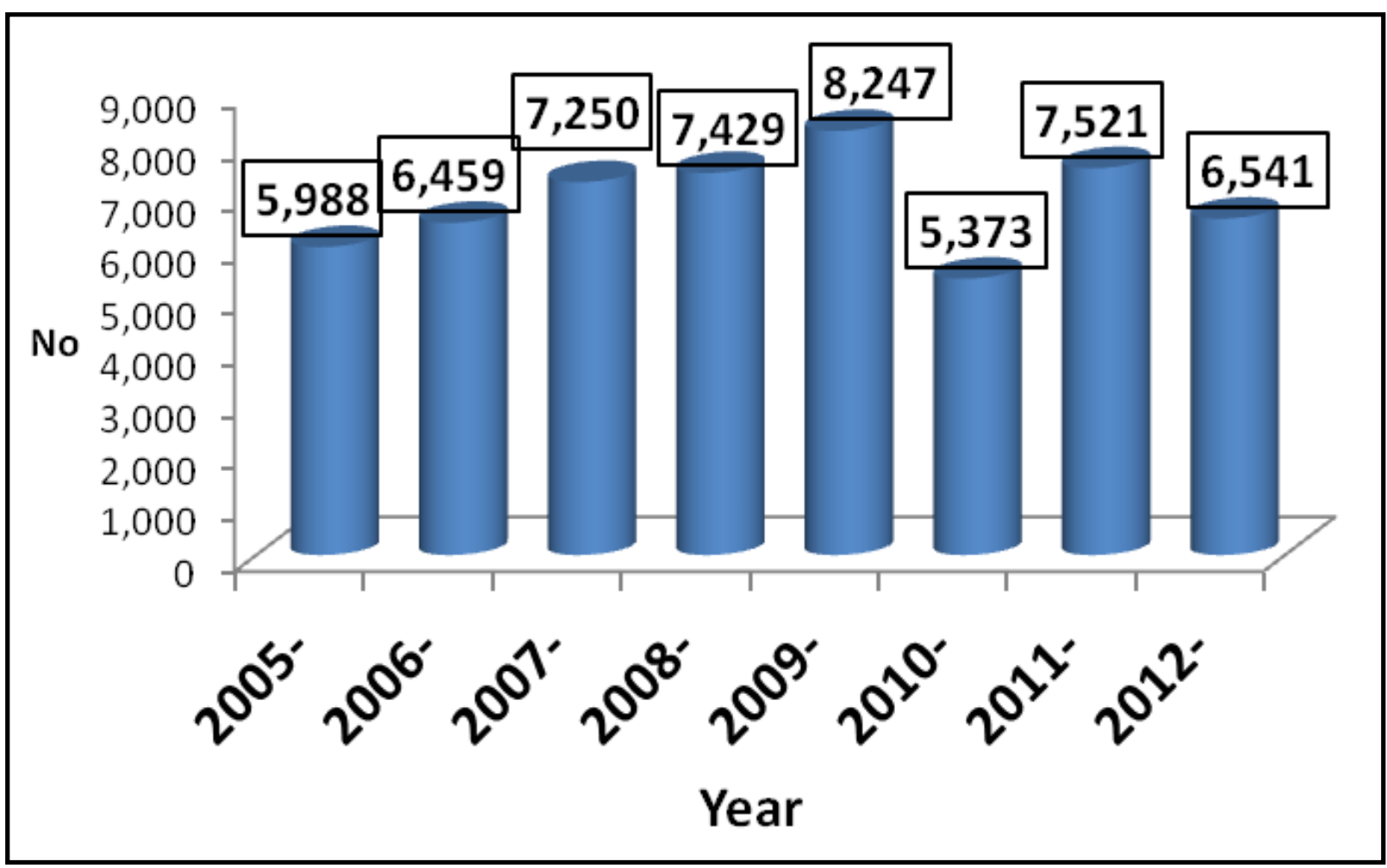

Figure (4): No of Trauma Cases admitted to Trauma Unit, AUH from Assiut Governorate 2005-2012 
Table (8): Cause- Specific Mortality Rate for cases admitted to Trauma Unit, AUH 2005-2012

\begin{tabular}{|ll|c|c|c|}
\hline Type of injury & Total Cases & No. of deaths & Incidence Rate \\
\hline AB & 754 & 22 & $29 / 1,000$ \\
\hline- & BO & 6,372 & 175 & $27 / 1,000$ \\
\hline- & FAI & 3,068 & 172 & $\mathbf{5 6 / 1 , 0 0 0}$ \\
\hline- & FG & 21,750 & 743 & $\mathbf{3 4 / 1 , 0 0 0}$ \\
\hline- & FH & 11,627 & 440 & $\mathbf{3 8 / 1 , 0 0 0}$ \\
\hline- & IPV & 6,402 & 227 & $\mathbf{3 5 / 1 , 0 0 0}$ \\
\hline- & MI & 1,598 & 45 & $28 / 1,000$ \\
\hline- & RTA & 23,534 & 1,261 & $\mathbf{5 4 / 1 , 0 0 0}$ \\
\hline- & SI & 2,217 & 40 & $18 / 1,000$ \\
\hline
\end{tabular}

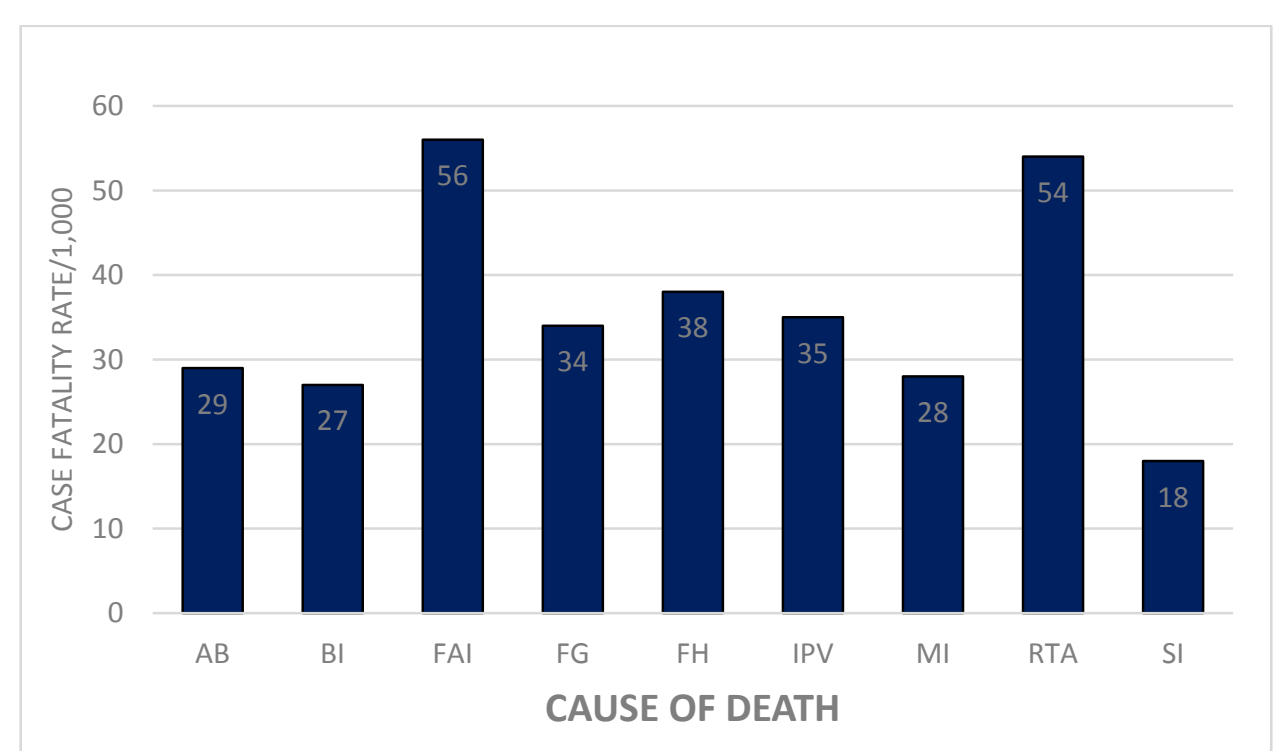

Figure (5): Cause-Specific Mortality Rate for Cases admitted to Trauma Unit, AUH 2005-2012

Table (9): The distribution of injuries in the body parts for cases admitted to Trauma Unit, AUH 2005-2012

\begin{tabular}{|l|c|c|}
\hline \multicolumn{1}{|c|}{ Body parts } & No. & \% \\
\hline Head & 12718 & $\mathbf{1 6 . 4}$ \\
\hline Upper limb & 18523 & $\mathbf{2 3 . 9}$ \\
\hline Lower limb & 20104 & $\mathbf{2 6 . 0}$ \\
\hline Abdomen & 3249 & 4.2 \\
\hline Chest & 4257 & 5.5 \\
\hline Pelvis & 3443 & 4.4 \\
\hline Vertebral column & 4764 & 6.2 \\
\hline
\end{tabular}




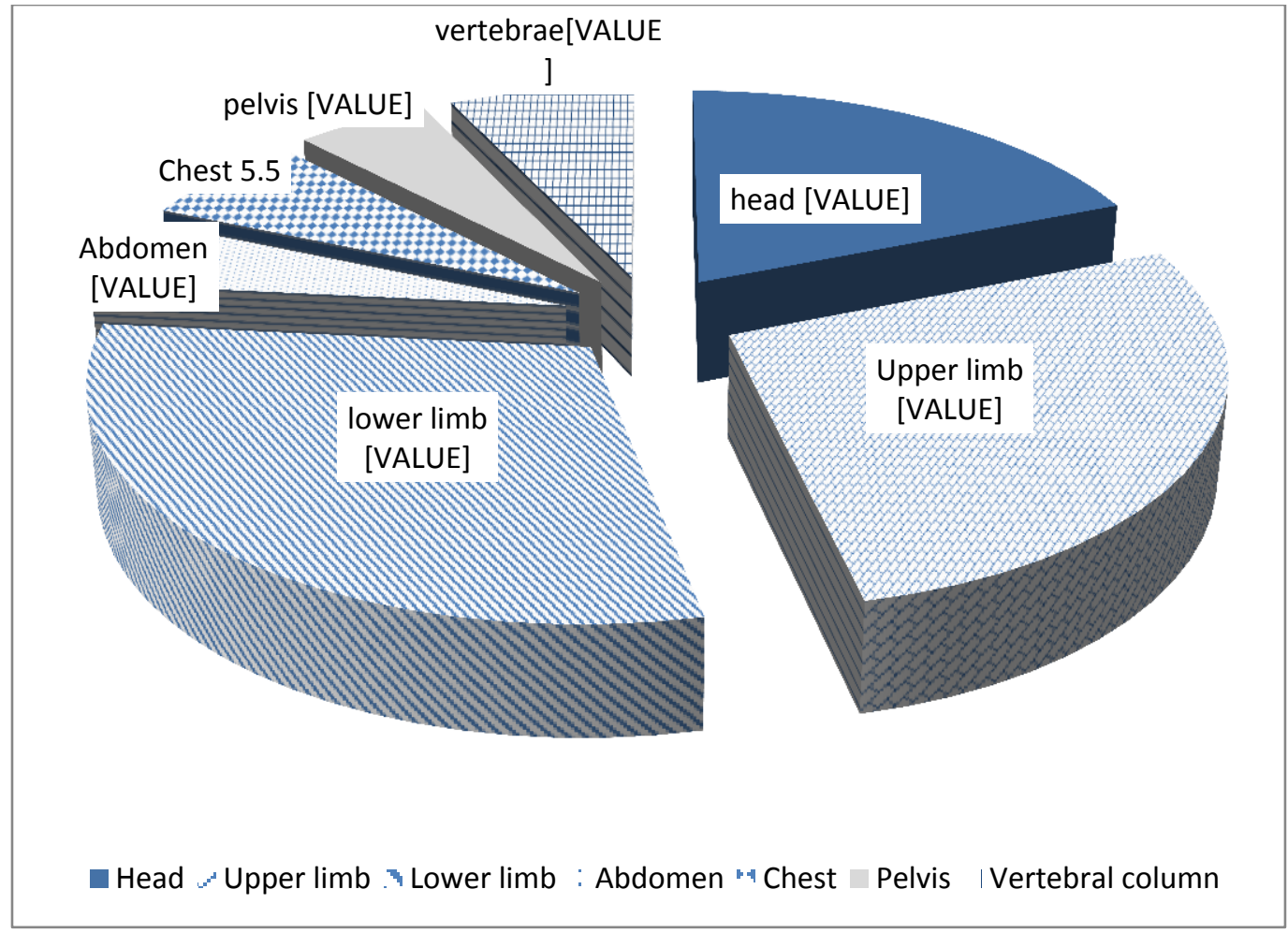

Figure (6): Distribution of injuries on body parts in cases admitted to Trauma Unit, AUH 2005-2012

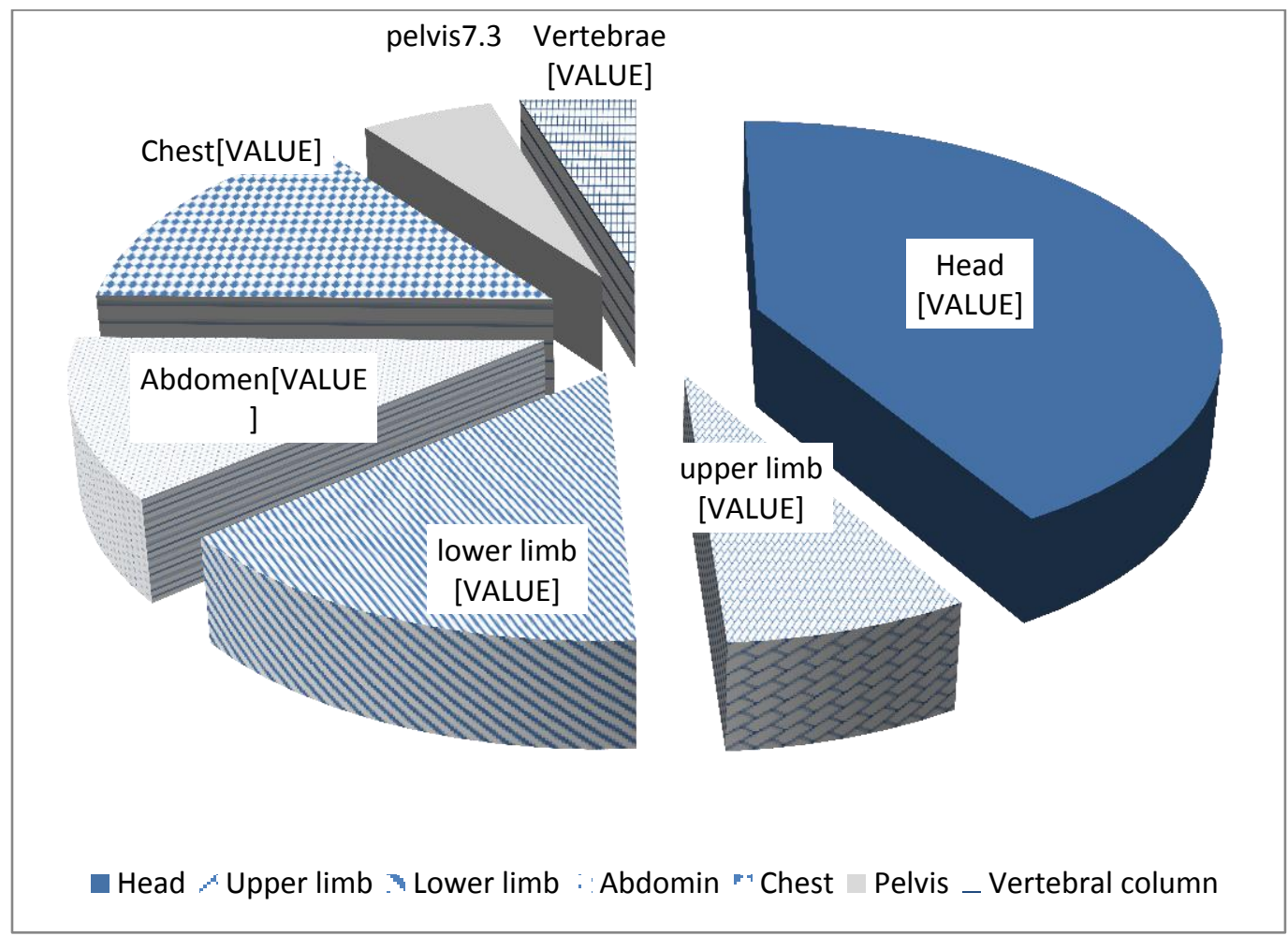

Figure (7): Distribution of injuries in body parts in mortality cases admitted to Trauma Unit, AUH 2005-2012. 
Table (10): Distribution of mortality cases according to the injured body area and cause of the death of cases admitted to Trauma Unit, AUH 2005-2012

\begin{tabular}{|c|c|c|c|c|c|c|c|c|c|c|c|c|c|c|c|c|}
\hline \multirow[t]{2}{*}{ Cause } & \multicolumn{2}{|c|}{$\mathbf{A B}$} & \multicolumn{2}{|c|}{ BO } & \multicolumn{2}{|c|}{ FAI } & \multicolumn{2}{|c|}{ FG } & \multicolumn{2}{|c|}{ FH } & \multicolumn{2}{|c|}{ IPV } & \multicolumn{2}{|c|}{ MI } & \multicolumn{2}{|c|}{ RTA } \\
\hline & No & $\%$ & $\mathrm{No}$ & $\%$ & No & $\%$ & $\mathrm{No}$ & $\%$ & No. & $\%$ & No. & $\%$ & N. & $\%$ & $\mathrm{No}$ & $\%$ \\
\hline $\begin{array}{l}\text { Head } \\
(\mathrm{n}=1798)\end{array}$ & 6 & 0.3 & 41 & 2.3 & 110 & 6.1 & 93 & 5.2 & 284 & 15.8 & 128 & 7.1 & 17 & 0.9 & $\begin{array}{c}111 \\
9\end{array}$ & 62.2 \\
\hline $\begin{array}{l}\text { Upper } \\
\text { limb } \\
(n=313)\end{array}$ & 0 & 0.0 & 5 & 1.9 & 35 & 11.1 & 0 & 0.0 & 93 & 29.6 & 23 & 7.3 & 0 & 0.0 & 157 & 50.0 \\
\hline $\begin{array}{l}\text { Lower } \\
\operatorname{limb} \\
(n=638)\end{array}$ & 0 & 0.0 & 12 & 1.9 & 75 & 11.8 & 168 & 26.3 & 29 & 4.5 & 0 & 0.0 & 12 & 1.9 & 342 & 53.6 \\
\hline $\begin{array}{l}\text { Abdomen } \\
(\mathrm{n}=499)\end{array}$ & 0 & 0.0 & 12 & 2.4 & 186 & 37.2 & 6 & 1.2 & 41 & 8.2 & 17 & 3.4 & 6 & 1.2 & 231 & 46.4 \\
\hline $\begin{array}{l}\text { Chest } \\
(\mathrm{n}=632)\end{array}$ & 0 & 0.0 & 6 & 0.9 & 58 & 9.2 & 23 & 3.6 & 133 & 21.0 & 0 & 0.0 & 0 & 0.0 & 412 & 65.2 \\
\hline $\begin{array}{l}\text { Pelvis } \\
(\mathrm{n}=232)\end{array}$ & 6 & 2.6 & 6 & 2.6 & 6 & 2.6 & 12 & 5.2 & 52 & 22.3 & 0 & 0.0 & 6 & 2.6 & 145 & 62.2 \\
\hline $\begin{array}{l}\text { Vertebral } \\
\text { column } \\
(\mathrm{n}=203)\end{array}$ & 0 & 0.0 & 6 & 2.9 & 0 & 0.0 & 12 & 5.9 & 69 & 34.3 & 0 & 0.0 & 0 & 0.0 & 116 & 56.9 \\
\hline
\end{tabular}

\section{DISCUSSION}

This study provided an important knowledge to identify the common causes of traumatic morbidity and mortality in Upper Egypt district. This knowledge aimed also, to evaluate the health services provided by the tertiary trauma center in AUH which presented by the highest qualified staff members, as well as evaluation of the documentation system used and point to the defects.

The current study examined the database of the AUH trauma center in the period from 2005 to 2012. After this period, from 2013 up till now the data base was not involved in our study because; new regulations limited the admission to AUH to three days a week only so affecting the rate admission.

In the current study, 77,380 cases admitted to the AUH trauma unit. The highest admission was in 2009 (8247cases/year) the incidence rate was 227/ 100,000 compared to the lowest admission in 2010 (5373 cases/year) with an incidence rate 145/100,000. This difference in admission rate could not be explained.

The age range of the admitted patients was 0-105 years with mean of 29.41. Most of the injured patients were under 30 years $(60.4 \%)$ which coincide with the WHO report of Egyptian injury surveillance (2010) where highest number of these deaths and injuries occurs among men in the age group 15-44 years.

Male: female ratio was $3: 1$ in this study which is in agree with Saudi population in Bakarman and Njaifan study (2014) and Nigerian population in Thanni and Kehinde study (2006). This could be explained by the fact that males involved more in life outside activities where they are exposed more than females to traumatic injuries.

In World Health Organization demographic, social and health indicator for Egypt unemployment rate was 12\% in 2012 (World Health Organization, 2013). 
In the present study, $60 \%$ of the admitted patients to trauma center was unemployed, proving that Upper Egypt is a poor region in Egypt compared to other regions in Egypt.

Mortality rate in the study was $4 \%$ compared to Thanni and Kehinde (2006) study $2.1 \%$. In the report of Egyptian injury surveillance 2010, nearly $20 \%$ of injured persons were hospitalized and deaths in the emergency room accounted for 1-2\% of total deaths (World Health Organization, 2010). The high incidence of mortality rate in this study owed to; the fact that AUH covers all Upper Egypt with high admission rate and the cases may be referred from other distant hospitals taking long time to reach the hospital. However; this result should be alarming to the hospital authorities to take corrective measures to decrease the mortality rate. Krug et al. (2002) stated that the rate of violence related death in low- to middle-income countries was more than twice that in high-income countries, although rates vary between regions and within countries.

Study conducted in Alexandria, Egypt by Wahab (2012), reported that the injury-related deaths were $69.2 \%$ in the age period between 15 to 60 years and $18 \%$ of deaths were in children less than 15 years of age, this in agreement with this study, where most of deaths occur under 30 years $(51.3 \%)$.

In the current study, fall both on ground $\mathrm{FG}$ and from height $\mathrm{FH}$ considered as the first and third causes of admission and mortality in patients admitted to AUH and both accounts for $43.1 \%$ of the total admitted cases and lead to death in $37.9 \%$ of traumatic deaths. FG and FH occur in 41.7 and $44.8 \%$ (respectively) of cases under 20- years age. Mortality rate in $\mathrm{FH}$ in children under 10 years was $37 \%$ followed by $14.4 \%$ in $60-70$ years. Lower limbs are the main body region affected by fall followed by the vertebral column injuries. These results coincide with the report of Egyptian injury surveillance 2010 where fall considered as the second cause of trauma (World Health Organization, 2010), as they did not split types of fall as we did in this study. In Wahab (2012) study fall was the fourth leading cause $(9.9 \%)$ of injury-related deaths, the burn cases were admitted to the trauma center and there was a great percent of drowning deaths.

In our study, RTA is considered as the second cause of injury $(30.4 \%)$ and deaths $(40.2 \%)$. Mortality occur in the age under 10 years by $29.6 \%$. Affection of the chest, abdomen and head are more common. These results coincide with; Patil et al. (2008) study on Indian population, report of Egyptian injury surveillance (2010) and Wahab (2012). Previous study done in AUH, the RTA constitutes $25 \%$ of the admission cases in 1999 (Abdel Hady and Abdel Moneim, 2001) this indicate the increase in the rate of these injuries this may return to the increase in the population and to other causes should be investigated. In Brazilian study, they found children 6-12 years are the most predominant in road traffic accidents (Figueiredo et al., 2011). The head followed by the lower limbs injuries were the most common injuries in RTA in the current study, this consistent with Abdel Hady and Abdel Moneim study (2001).

Firearm injuries in this study represent $4 \%$ of admitted cases. These injuries increased across the study time to become the fourth cause of 
admission (10.5\% of cases) and the second leading cause of death in 2012. This change in the trend of injury causes could be explained by the country security instability after revolution 2011 and the easy availability of weapons. In pervious study done by Mahran et al. (2016) in AUH from 2002 to 2009, the FAI were ranging between the sixth to the ninth cause of injury meaning that FAI is an increasing problem in Upper Egypt and should be faced by the authorities. The cause specific mortality rate in FAI was the highest $(56 / 1,000)$. The firearm injuries occurred predominantly in the age 10-30 years. Mortality occur under 10 years in $38.4 \%$ of cases. In American population, young adults aged 25- 34 years have the highest rate of fatal firearm injury $(15.1$ per $100,000)$ of all age groups, followed by those in the 15 to 24-year age (14.4) (Fowler et al., 2015).

Although many data were obtained but still there were many limitations we met during this study including; i) missing data, ii) no details about the position of the injured patients in RTA whether occupants, drivers or pedestrians, iii) no universal definition of the types of injuries like ICD-10, iv) no documentation of the injury severity according to any injury severity scales, and v) the current documentation was missing many details of medicolegal importance e.g. type of weapon caused the injury in firearm injuries.

\section{RECOMMENDATIONS}

Preventive measures should be taken to decrease the mortality rate. This could be done by using any of the injury severity scales to evaluate to the patients and identifying patients at risk. Using uniform definitions of the injuries to facilitate documentation and survey performance. Detailed studies for every cause of injury should be done for more benefits.

\section{CONCLUSION}

This study concludes that road traffic accidents, falls and interpersonal violence are the main causes of injuries and deaths. Attention should also be given to young patients who are exposed to these injuries, especially when the head, lower limb, chest or abdomen is injured. Attention must be paid to the detailed medical record of injuries for its medicolegal importance.

\section{REFERENCES}

Abdel Hady, R.H. and Abdel Moneim, W.M. (2001):

Medicolegal aspects of road traffic accidents in Assiut university hospital. Egypt. J. Forensic Sci. Appl. Toxicol., 1: 88-98.

Bakarman, $M$. and Njaifan, $\mathbf{N}$. (2014): Assessment of nonemergency cases attending emergency Department at King Fahad General Hospital, Jeddah; Pattern and Outcomes. Life Sci. J., 11(8): 20-5. Available at: http://www.lifesciencesite.com [Accessed at October 2015].

Dsouza, C.; Rao, V., Kumar, A. and Diaz, E. (2014): Epidemiological Trends of Trauma in Tertiary Care Centre in Dakshina Kannada District of Karnataka, India. J Clin Diagn Res., 8(3): 66-68.

Figueiredo, I.; de Carvalho, M.V. and de Lima, G.M. (2012): Pediatric trauma due to motor vehicle accidents on high traffic roadway. Einstein (Sao Paulo), 10(1):29-32. 
Fowler, K.A.; Dahlberg, L. L.; Haileyesus, T. and Annest, J. L. (2015): Firearm injuries in the United States. Prev. Med., 79: 514.

Holder, Y.; Peden, M.; Lund, J.; Gururaj, G. and Kobusingye, O., Ed. (2001): Injury surveillance guidelines. $1^{\text {st }}$ ed. Geneva, World Health Organization. pp.5-6. Available at:

http://www.who.int/violence_injury_pr evention/violence/world_report/en/. [Accessed at October 2015].

Kovacs, G. and Croskerry, P. (1999): Clinical decision making: an emergency medicine perspective. Acad. Emerg. Med., 6 (9):947-52.

Krug, E.G.; Dahlberg, L. L.; Mercy, J. A.; Zwi, A.B. and Lozano, R., Ed. (2002): World report on violence and health. Geneva, World Health Organization. Available at: www.who.int/violence_injury_prev ention/violence/world_report/.../intr oduction.pdf [Accessed at May 2015]

Mahran, D.; Farouk, O.; Qayed, M. and Berraud, A. (2016): Pattern and Trend of Injuries Among Trauma Unit Attendants in Upper Egypt. Trauma Monthly, 21(2): e20967.

Patil, S. S.; Kakade, R.; Durgawale, P. and Kakade, S. (2008): Pattern of road traffic injuries: a study from Western maharashtra. Indian $\mathbf{J}$ Community Med., 33(1):56-7.
Recktenwald,

K.; Hunsaker, D.M.; Corey, T. S. and WeakleyJones, B. (2005): Clinical forensic medicine introduction for healthcare providers. J Ky Med Assoc., 103(9):433-5.

Runyan, C.W.; Bowling, J.M. and Bangdiwala, S.I. (1992): Emergency department record keeping and the potential for injury surveillance. J Trauma, 32(2):1879.

Sharma, B.R. (2006): Clinical forensic medicine in the present-day trauma-care system--an overview. Injury, 37(7):595-601.

Thanni, L.O. and Kehinde, O.A. (2006): Trauma at Nigerian teaching hospital: pattern and documentation of presentation. Afr. Health Sci., 6 (2): 104-107.

Wahab, M.M. (2012): Incidence and patterns of injury-related mortality: a register-based follow-up study in Alexandria, Egypt (2000-2010). J Egypt Public

Health Assoc., 87(1-2):8-15.

Wegman, B.; Stannard, J.P. and Sonny Bal, B. (2012): Medical Liability of the Physician in Training. Clin Orthop Relat Res., 470(5): 1379-85.

World Health Organization (2007): Preventing Injuries and Violence. A Guide for Ministries of Health Available at: www.who.int/violence_injury_prev ention/publications/injury.../preven tion_moh/en/ (Accessed at April 2014).

World Health Organization (2010): World Health Organization Injury surveillance: a tool for decisionmaking: annual injury surveillance report, Egypt, 2009. Egypt: Regional Office for the Eastern 
Mediterranean, Ministry of Health; 2010. Available from: http://www.emro.who.int/dsaf/dsa1 087.pdf. [Accessed at October 2015].

World Health Organization (2013): Demographic, Social and Health Indicator for Countries of the East Mediterranean. Available at: http://www.applications.emro.who. int/dsaf/EMROPUB_2013_EN_15 37.pdf [Accessed at October 2015]. Yu, K.T. and Green, R.A. (2009): Critical aspects of emergency department documentation and communication. Emerg. Med. Clin. North Am., 27(4):641-54 


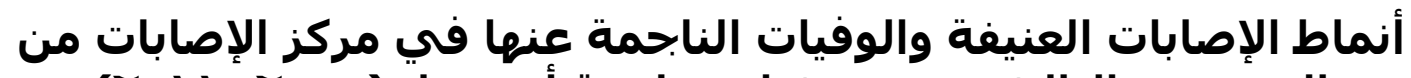

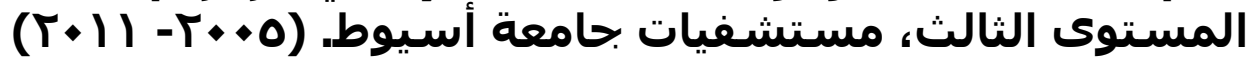

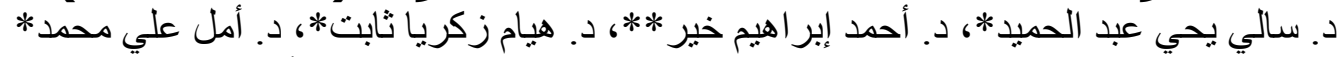

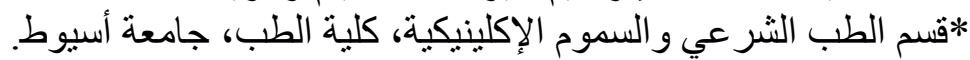

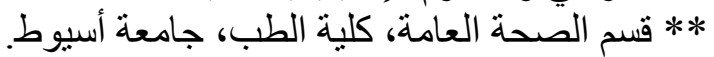

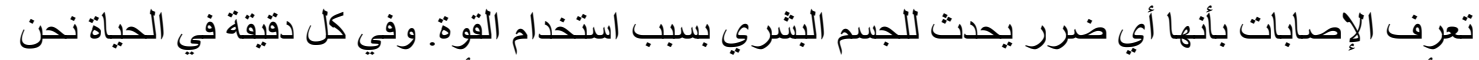

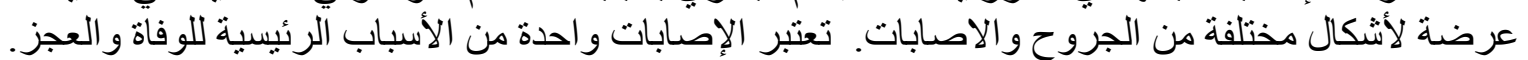

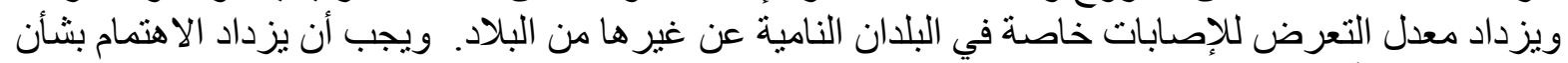

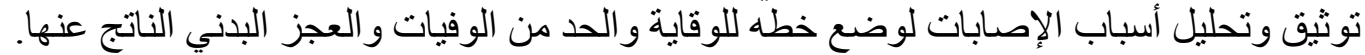

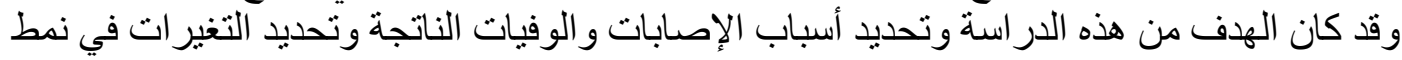

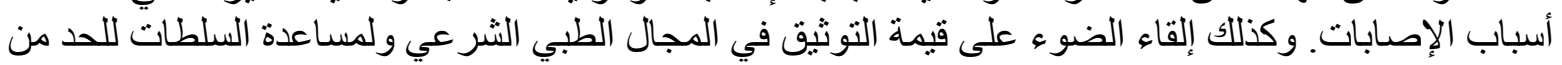
هذه الدر اسة در اسة وصفية رجعية حيث أجريت على المرضى المصابين المحجوزين في مركز الإصابات

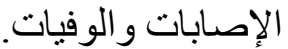

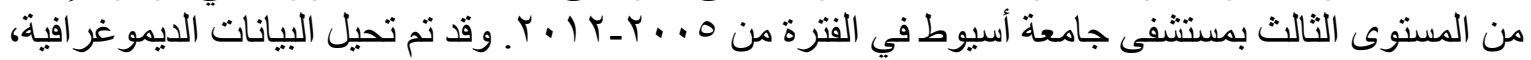

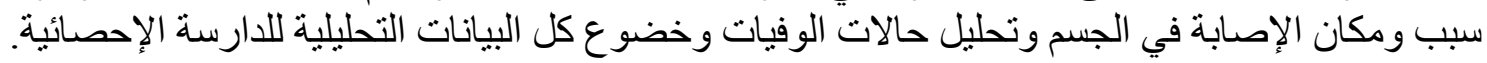

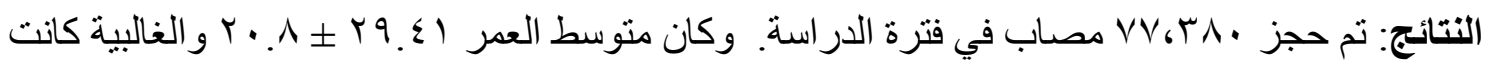

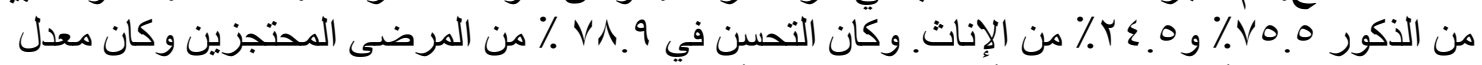

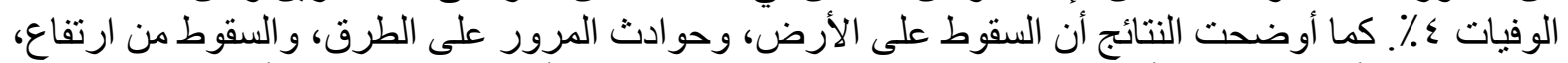

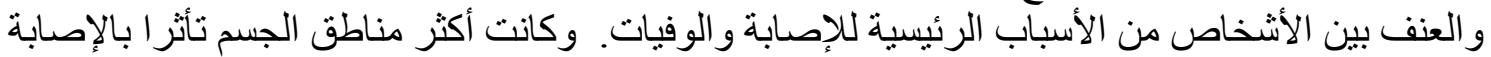
الأطر اف السفلية والأطر اف العليا والر أس والعمود الفقري والصدر والعة والحوض الأكثر تليهم منطقة البطن.

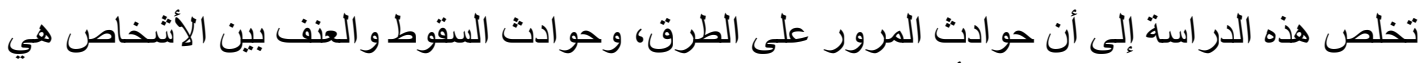

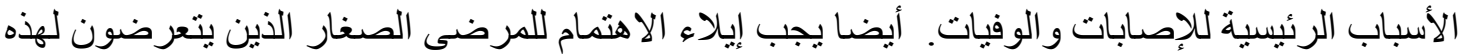

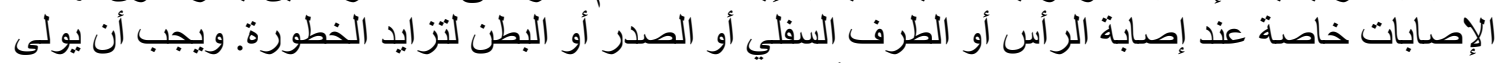
الاهتمام بالتسجيل الطبي التفصيلي للإصابة الصات لأهميته الطبية الثر عية. 